\title{
Use Circulating microRNAs as Biomarkers of Drug-Induced Liver Injury
}

\section{Xi Yang}

National Center for Toxicological Research, Jefferson, Arkansas, USA

Drug-Induced Liver Injury (DILI) is an adverse event that frequently leads to cessation of drug testing in clinical trials, restrictions on drug use, and drug withdrawals. Among the preclinical candidate compounds that are discontinued for organ toxicity, it is estimated that up to half are terminated due to hepatotoxicity. Existing biomarkers of liver injury (e.g., serum alanine aminotransferase [ALT] and bilirubin) provide reasonable indicators of damage; however, none of them has sufficient specificity and/or they are increased only after significant damage has occurred. New reliable biomarkers of DILI are urgently required for both clinical and preclinical pharmaceutical evaluation. Open access helps authors, who discover new DILI biomarkers, to maximize the impact of their research articles.

miRNAs are short ( $\sim 21$ to 25 nucleotides in length), single-stranded, non-coding RNA that were first found in Caenorhabditis elegans and are now recognized as novel agents exercising post-transcriptional control over most eukaryotic genomes. miRNAs are highly conserved among species, ranging from worms to humans, revealing their very ancient ancestry. It has been reported that miRNAs are expressed in all animal cells and have fundamental roles in cellular activities, such as development, cellular differentiation, proliferation, apoptosis, cell-cycle control, metabolism and cancer. It is estimated that $\sim 60 \%$ of human mRNAs are regulated by miRNAs [1], suggesting that miRNAs might very well form another layer of the regulatory circuitry that exists in the cell. Similar to mRNA, some miRNAs are produced in cell- or tissuespecific manners. Recently, many reports concerning miRNAs related to disease have been published and suggest that miRNAs may serve as a new kind of biomarker for organ injury.

Large numbers of miRNAs have been observed outside of cells of both healthy and diseased patients and animals. Recent studies indicate that miRNAs are actively released from cells via several different processes; however, the function of circulating miRNAs are unclear even though some studies have indicated that they can function in cellto-cell communication and regulation. miRNA in body fluids holds a unique position for use as a biomarker due to its unique stability. Unlike mRNA, miRNA has been shown to be remarkably stable in many different body fluids, including blood and urine. This stability greatly facilitates its use as a clinical biomarker of disease and injury since sample handling and processing are much less problematic when compared to mRNA. miRNA-based biomarkers also have many advantages over protein-based biomarkers primarily due to the fact that miRNA is a relatively simple molecule that can be detected using standard, robust molecular biology techniques.

Using an acetaminophen-induced mouse model of DILI, Wang et al. reported that the level of many plasma miRNAs inversely correlated with the level of hepatic miRNAs, indicating that for these miRNAs, hepatic injury caused the release of miRNAs into the circulation [2]. Specifically, miRNA-122 and miRNA-192, which are predominantly expressed in the liver, increased in the plasma with concurrent decreases in the liver. The increases in both miRNAs were detected earlier than the increase of ALT. The increase of serum miRNA-122 and miRNA-192 was confirmed recently in patients with acetaminophen poison; Yang et al. recently reported that urinary miRNA profiles were altered in rats after administration of hepatotoxic doses of acetaminophen or carbon tetrachloride [3,4]. The level of the same 10 urinary miRNAs was increased in acetaminophen and carbon tetrachloride treated rats. In addition, the miRNA expression profiles were able to distinguish the patterns of the responses to the two hepatotoxicants vs. a nonhepatotoxicant (penicillin) and vehicle controls.

Although miRNA detection is theoretically simpler and subject to fewer confounders than developing protein based assays, there are some technical issues associated with miRNA measurement. One great concern is interlaboratory variability in the measurement of miRNAs, particularly when different platforms are used. RT-qPCR and microarrays are the two commonly used platforms for global miRNA detection; Next-Generation sequencing is another new method based on direct sequencing. Unfortunately, at this time, there appears to be limited correlation between the different platforms, most likely due to the strong influence of different primer designs and sequence heterogeneity. Lack of standard protocols for sample preparation also makes comparisons between studies difficult. In contrast to total RNA, assessment of miRNA quantity and quality is less than ideal. Finally, and especially for cell-free miRNA analysis, a variety of different normalization processes have been used to control for technical and biological variability but none of them are ideal.

\section{References}

1. Friedman RC, Farh KK, Burge CB, Bartel DP (2009) Most mammalian mRNAs are conserved targets of microRNAs. Genome Res 19: 92-105.

2. Wang K, Zhang S, Marzolf B, Troisch P, Brightman A, et al. (2009) Circulating microRNAs, potential biomarkers for drug-induced liver injury. Proc Natl Acad Sci USA 106: 4402 - 4407

3. Yang X, Greenhaw J, Shi Q, Su Z, Qian F, et al. (2012) Identification of urinary microRNA profiles in rats that may diagnose hepatotoxicity. Toxicol Sci 125 335-344.

4. Yang X, Li Z, Su Z, Davis K, Chen T, et al. (2011) Urinary microRNAs as noninvasive biomarkers for acetaminophen-induced liver injury. J Postgenom Drug Biomark Develop 1: 101.

Corresponding author: Xi Yang, US FDA NCTR, 3900 NCTR Road, Jefferson, Arkansas 72079, USA, Tel: (870) 543-7321; E-mail: xi.yang@fda.hhs.gov

Received June 29, 2012; Accepted June 29, 2012; Published July 03, 2012

Citation: Yang X (2012) Use Circulating microRNAs as Biomarkers of DrugInduced Liver Injury. J Vaccines Vaccin 3:e105. doi:10.4172/2157-7560.1000e105

Copyright: (c) 2012 Yang X. This is an open-access article distributed under the terms of the Creative Commons Attribution License, which permits unrestricted use, distribution, and reproduction in any medium, provided the original author and source are credited. 